\title{
Carnets
}

Revue électronique d'études françaises de l'APEF

Deuxième série - 3 | 2015

Insulaire

\section{Une île bizarrement habitée}

Natália Guilhermina Oliveira Lameiras Alves

\section{(2) OpenEdition}

\section{Journals}

Édition électronique

URL : http://journals.openedition.org/carnets/1424

DOI : $10.4000 /$ carnets. 1424

ISSN : 1646-7698

Éditeur

APEF

\section{Référence électronique}

Natália Guilhermina Oliveira Lameiras Alves, « Une île bizarrement habitée », Carnets [En ligne], Deuxième série - 3 | 2015, mis en ligne le 28 février 2015, consulté le 02 mai 2019. URL : http:// journals.openedition.org/carnets/1424; DOI : 10.4000/carnets. 1424

Ce document a été généré automatiquement le 2 mai 2019.

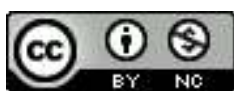

Carnets est mis à disposition selon les termes de la licence Creative Commons - Atribution - Pas d'utilisation commerciale 4.0 International. 


\title{
Une île bizarrement habitée
}

\author{
Natália Guilhermina Oliveira Lameiras Alves
}

1 Dans le conte L'T̂le aux oiseaux de fer, nous nous apercevons que Dhôtel concède aux descriptions de ses espaces une vraisemblance qui fait qu'ils se trouvent proches des espaces réels. Ceci lui permettra dans le récit de maitriser ses personnages, ainsi que leurs mouvements. De ce fait, en tant que lectrice, nous nous déplaçons, aux côtés des protagonistes, dans un monde quasiment réel et les suivons sans que nous nous sentions dépaysés. Nous vérifions donc que «À travers la disjonction des lignes, comme par le dédoublement des contours, un intervalle s'insinue dans le paysage, il le transforme en lieu d'étrangeté, qui attire le regard en excluant le point de vue » (Ropars-Wuilleumier, 2002 : 47). Marianna Antonnella Todero affirme à ce propos que « Dhôtel, par sa capacité de superposer l'ici et l'ailleurs peut être catalogué parmi "ces magiciens du regard, qui dans le banal quotidien, s'acharnent à ouvrir des perspectives autres" » (Todero, 2006 : 132).

2 En effet, il existe une esthétique singulière autour du domaine de l'espace, étant donné que les descriptions des lieux nous sont faites par le biais de l'observation des personnages, les différents décors surgissant au fur et à mesure que s'effectue leur quête et selon les itinéraires qu'ils suivent. Ces lieux aux dimensions presque humaines sont sillonnés la plupart du temps par des personnages à pied (cf. Blondeau, 2003: 257), qui nous offrent une vision plus détaillée des lieux parcourus. La toponymie, à son tour, aide, d'une part, à susciter l'illimité, mais, d'autre part, à situer l'action dans un espace qui se veut concret. Effectivement, le silence qui émane de ces lieux peut être vu comme un prélude à l'aventure puisqu'il favorise le rêve d'un ailleurs du personnage principal.

3 Il nous faut rappeler un fait important, à savoir qu'André Dhôtel était surnommé «le promeneur campagnard » (Blondeau, 2006 : 95). En effet, il prenait plaisir à se promener sans destination, à être surpris par le hasard des chemins, sans la contrainte de suivre un itinéraire défini au préalable. En harmonie avec la nature, il prenait le temps d'observer chaque détail, chaque espace qui s'offrait à ses yeux. Ses personnages reflètent, eux aussi, cet état d'esprit et, lorsqu'ils partent en promenade, ils ne se soucient ni de l'itinéraire à suivre ni du lieu d'arrivée. 
4 De plus, si nous nous attardons sur le titre du conte, nous vérifions, dès lors, qu'il annonce une localisation: une île habitée par des oiseaux de fer. En effet, pour Dhôtel, l'espace est le point de départ de toute fiction romanesque, les «lieux privilégiés se présent[a]nt comme des étapes d'où l'on repart » (Reumaux, 1979: 75). En reprenant ses expériences de vie et surtout les lieux de son enfance, Dhôtel définit au préalable les itinéraires imaginaires de ses récits et il dit, lui-même, à ce propos :

Une grande partie du travail consiste à tracer des itinéraires sur des cartes géographiques imaginaires. Si la topographie joue un si grand rôle, c'est d'abord parce que j'ai beau inventer des histoires, leur point de départ en est toujours fourni par un lieu réel que je me contente par la suite de transposer. (Apud Blondeau, 2003 : 233).

5 En somme, l'espace dhôtelien peut être, d'une part, riche et multiple et, d'autre part, de par son essence réaliste même, restreint et limité, et les personnages, ainsi que le lecteur, ne se sentent nullement perturbés lorsqu'ils basculent dans l'un ou l'autre de ces lieux. En ce qui concerne l'espace du récit, Jean Weisgerber, dans son ouvrage L'espace romanesque, mentionne que :

L'espace du récit, enfin, est vécu à différents niveaux : d'abord par le narrateur en tant que personne physique, fictive ou non, et à travers la langue qu'il utilise; ensuite, par les (autres) personnages qu'il campe ; en dernier lieu, par le lecteur qui introduit à son tour un point de vue éminemment partiel (Weisgerber, 1978 : 13).

Les décors que nous plante le narrateur aident donc à situer les héros dans sa quête, mais les minutieuses références spatiales aident également à définir le caractère, à déterminer le comportement et les sentiments de ces personnages. "Plutôt que sur la société, l'univers d'André Dhôtel est fondé sur des sociétés. Pour cet écrivain si attentif aux plantes et aux insectes, les hommes se définissent également par leur milieu et les règles sociales dépendent aussi de l'espace et de l'environnement» (Blondeau, 2003: 176). Voyons donc comment l'écrivain procède à cela dans le conte L'île aux oiseaux de fer et partons à la recherche d'une de ses autres passions, à savoir, la mer.

\section{Comme une bouteille à la mer}

7 Le thème de l'eau, et plus précisément l'espace de la mer, occupent une place très marquante dans les œuvres de cet écrivain. L'élément aquatique prend diverses formes, à savoir, celle d'une rivière, d'un étang, d'un viaduc, d'un canal et même, de la pluie. Ainsi, et parce que dans les Ardennes lacs, étangs, fleuves, rivières, pluie abondent, l'eau est source de vie pour Dhôtel, mais aussi raison de tension et de déséquilibre.

8 L'eau, parce que c'est un élément à la fois rassurant et menaçant, apparait de manière récurrente dans les récits d'initiation. C'est pourquoi, dans L'île aux oiseaux de fer, l'auteur jette son héros dans l'océan, car l'eau est le symbole de l'initiation à la vie, de la purification (lors de la cérémonie de baptême) mais également du recommencement, du renouveau. Épreuve à surmonter, puisque le héros peut être englouti par les vagues, la mer est également une étape à atteindre, dans la mesure où elle est aussi l'objet d'un nouveau départ.

9 Ainsi, le héros dhôtelien se retrouve jeté en plein océan, tout comme une bouteille que l'on lance à la mer dans le but de pouvoir être trouvée par quelqu'un. En permettant que son héros chavire en haute mer, Dhôtel semble vouloir lancer un appel au secours, dans l'espoir que le lecteur l'entende, comme s'il s'agissait d'une invitation à l'aventure. Mais 
cet événement doit surtout être vu comme un défi que le héros doit surmonter au long de sa quête.

L'auteur semble vouloir nous orienter géographiquement et souligne donc que « le bateau suivait une route merveilleuse (...). Il descendit vers le sud, emboucha le détroit de Gibraltar et se dirigea vers Alexandrie » (Dhôtel, 2002 : 20) ; qu'il « avait abordé à Ceylan, dans un port de l'Inde et à Java, puis au long des jours il fila à travers le Pacifique (...) » (Dhôtel, 2002 : 20). Dhôtel nous dessine une carte, nous guide dans l'immensité de l'océan, pour que nous ne nous perdions pas, pour que nous ne nous sentions pas dépaysés. Toutefois, et contrairement aux autres espaces dhôteliens, tels que les routes, les villes et même les forêts, en mer l'orientation est difficile, il n'y existe aucun point de repère.

Par conséquent, dans L'île aux oiseaux de fer, l'eau revêt un aspect particulier, elle est source d'un terrible danger et suscite donc une profonde crainte : Julien décide de partir à l'aventure et accepte de travailler sur un bateau de croisière, or, il est jeté par-dessus bord par celui qu'il pensait être son ami et l'eau surgit dans toute son immensité et devient, dès lors, peu rassurante: un "grand silence» (Dhôtel, 2002: 25) s'installe. Néanmoins, le lecteur s'apaise dès qu'il perçoit que la mer prend en charge la vie du héros; les pensées de Julien, ses émotions paraissent être comprises par la mer qui change le mouvement de ses vagues : le héros est « dévoré par la peur » (Dhôtel, $2002: 25$ ) et la mer devient «faiblement onduleuse " (Dhôtel, 2002 : 25), celle-ci ayant la fonction d'apaiser le héros et de l'accueillir dans son immensité.

Le narrateur ajoute de surcroît que la mer recueille les morts de l'île, et « les oiseaux les emportent vers la haute mer, et s'ils veulent revivre ils reviennent ici parmi nous » (Dhôtel, 2002 : 25). La mer enlève la vie, mais elle peut, également, la redonner, comme si l'eau de la mer avait un pouvoir surnaturel, celui de la résurrection. Nous imaginons la mer enveloppant les morts, témoignant de son affection et de sa protection, et jouant du mouvement des vagues pour les bercer.

Dans ce sens, nous pouvons ajouter que l'auteur personnifie la mer, puisqu'il lui confère des sentiments, qu'elle exprime, par exemple, au cours de la fuite des deux protagonistes, Julien et Irène : lorsqu'ils essaient de s'enfuir de l'emprise de l'île, « la mer se [soulève] en vagues énormes » (Dhôtel, 2002 : 115), comme si elle se révoltait contre eux, comme si elle voulait les empêcher de quitter cette île et les maintenir prisonniers de cet espace qu'elle protège. Révoltée, la mer devient un obstacle que les deux personnages dhôteliens doivent surmonter s'ils veulent atteindre le bonheur, la liberté. Ensemble, Julien et Irène combattent la furie de la mer, qui veut à tout prix les maintenir près d'elle, et, pour se libérer de cette oppression, ils doivent lutter et démontrer qu'ils méritent d'atteindre le bonheur.

Par conséquent, nous constatons que l'eau, et par là même la mer, éveille les sens du personnage dhôtelien. En effet, et selon Gaston Bachelard : « on est si loin de la terre, de la vie terrestre, que cette dimension de l'eau porte le signe de l'illimité » (Bachelard, 1957 : 186). Inquiétante, dangereuse et tout à la fois attirante, la mer représente un ailleurs, un espace symbolique qui mène à d'autres lieux, tels que, par exemple, lîle.

\section{Une Île déconcertante}

L'île de ce conte peut, par conséquent, être considérée un "espace maléfique qui va retenir [le héros] prisonnier et qu'il faudra fuir ou transformer» (Perry, 2006: 69). Cet 
espace délimité, cette terre entourée d'eau salée de tous côtés, fait que les occupants soient des prisonniers, des êtres vivant isolés du monde. Cet espace insulaire apparaît comme un espace symbolique, où les êtres sont gouvernés par des machines qui l'habitent et il rompt avec les décors auxquels Dhôtel nous avait habitués : les villages ardennais. Julien, le personnage principal, se retrouve, de la sorte, enfermé dans un espace ouvert, perdu dans un endroit limité et restreint, déstabilisé : «'Des oiseaux de fer', murmura Julien. D'abord Julien n'en crut pas ses yeux ni ses oreilles (...) » (Dhôtel, 2002: 28). « Ne nous étonnons pas trop, songea-t-il. Nous connaissons déjà toute cette mécanisation. Seuls les oiseaux... » (Dhôtel, 2002: 30). «Tout était si net en ce lieu que Julien Grainebis pressentait maints mystères » (Dhôtel, $2002:$ 49).

En effet, Dhôtel se munit de procédés créatifs et subtiles pour introduire l'insolite dans la diégèse, de sorte que le quotidien et l'imaginaire s'harmonisent naturellement, sans troubler le lecteur. Dans ce sens, il convient de rappeler que le monde «dhôtelland »concept décerné par Maurice Nadeau - est proche du quotidien. Toutefois, l'approche singulière qui est faite à ce dernier, fait en sorte que l'ingénieux mélange de réalisme et de fantaisie - l'intrusion de l'insolite - gère un monde autre. Dans ce sens, Charles P. Marie souligne que :

L'art d'André Dhôtel consiste à évoquer parallèlement et médiatement deux existences, la réelle et l'irréelle. Leur combinaison permet le dépassement de la raison. Il nomme cela « un pèlerinage » (« voyage où l'on ne se propose pas un but, mais une absence de but ») (Apud Cesbron, 1998 : 124).

Cependant, l'union entre ces deux univers - la réalité et la fantaisie - ne provoque aucunement chez le lecteur un sentiment d'angoisse ou de frustration; face au surgissement de l'insolite, le lecteur réagit naturellement, l'irréel pénétrant, en crescendo, dans le réel. Malgré le fait que l'étrangeté qui domine le conte se doive à la physionomie des oiseaux, qui sont tout droit sortis de l'imaginaire dhôtelien, il n'en demeure pas moins vrai que l'auteur parsème le décor du récit bref d'éléments oniriques afin d'intensifier l'univers fantaisiste. Par ailleurs, comme le réfère Hélène Védrine, "Alors que le symbolique se constitue dans le temps, l'imagination joue sur l'espace, sur le rapport du moi à l'image spéculaire » (Védrine, 1990 : 141).

Ce sentiment ambigu de surprise et d'appréhension gère, chez le lecteur, une envie de découvrir le mystère, l'insolite de cette île. En effet, contrairement à la forêt à laquelle Dhôtel nous avait habituée, où tout est sauvage, entouré d'arbres, de buissons, où l'on entend des oiseaux, où vivent des animaux, l'île, elle, n'a pour seul paysage que des cultures, des plantes qui permettent aux habitants de vivre. Rien d'autre n'y prend vie : aucun arbre, aucune herbe sauvage, aucun insecte, aucune espèce animale. Sur cette île «drôlement habitée " (Dhôtel, 2002: 23), toute vie, qu'elle soit végétale, animale ou humaine, doit être étudiée et autorisée au préalable par le gouvernement robotisé. "Julien regardait de tous ses yeux la campagne. Vaste défilé de cultures : cannes à sucre, dioscorées et d'autres plantes inconnues. Pas un arbre. A peu près aucune herbe sauvage. Pas de friches ni de lieux vagues" (Dhôtel, 2002: 52). Monique Venot Petitet nous fait observer que "Dans les romans d'André Dhôtel, le sentiment de l'absurde prend naissance au fil des pages, souvent dès la première, mais d'une manière si fugitive, si légère, qu'il peut passer inaperçu » (Venot Petitet, 1996 : 87).

Par ailleurs, nous constatons également qu'aucune appellation n'est donnée aux différents lieux de l'île. Nulle indication toponymique n'est fournie à la plage, à la ville, ou à la montagne, toutefois, l'espace se trouve parfaitement défini : « une série de boutons 
(...) desquels étaient inscrits les lieux de destination: Plage numéro I. Plage numéro II. Village. Montagne...» (Dhôtel, 2002: 56). Les indications de lieux sont déshumanisées, démunies de toute mémoire : toute présence du passé représentatif de l'humanité est effacée, c'est pourquoi, par exemple, nous ne trouvons pas de noms de rues portant le nom d'une personnalité, d'une date historique, d'une référence spatiale antérieure, etc. L'auteur se joue du récit traditionnel et, par le biais du point de vue du personnage, surprend le lecteur par la rupture avec le passé, par l'abolition des mémoires, des souvenirs commémoratifs de l'histoire de l'Homme et met en scène le présent, le progrès et la robotisation. Cette île est donc espace étrange, puisque l'homme y est démuni de tout sentiment, de toute affection.

Néanmoins, le lecteur continue à être surpris, puisque l'insolite est poussé à bout par son créateur, qui fait d'une île gouvernée par des robots un espace plaisant, les habitants y étant invités à se promener. Les individus peuvent se déplacer sur l'île, s'ils respectent rigoureusement les lieux auxquels ils ont le droit d'accès. En effet, « certaines régions de l'île demeuraient interdites [et] on ne savait pour quelles raisons » (Dhôtel, 2002 : 58). La montagne est, elle aussi, perçue comme espace interdit, au terrain infertile: « La végétation sauvage qui se développait le long des flancs abrupts était d'un vert assez sombre, sans grand attrait » (Dhôtel, 2002: 57). Sur cette île, la nature ne prend pas le dessus sur les robots, la beauté naturelle étant interdite aux hommes.

21 Il est, d'ailleurs, important de souligner que, sur cette île, d'autres lieux communs à Dhôtel s'y trouvent regroupés (à l'exception des chemins de fer) : campagne, montagne et ville y existent, mais sans beauté, sans attrait et sans humanité aux yeux du lecteur. «Dans ce lieu on distingu[e], d'un côté, l'ensemble de l'île, et de l'autre, les falaises et les forêts de la montagne. (...) Cultures, plages, bâtiments d'une longueur démesurée, isolés dans la campagne vers le sud, et, vers le nord, près de la mer, quelques maisons (...) » (Dhôtel, 2002 : 56-57).

Signalons que la nature dhôtelienne est chargée de symbolisme et qu'elle est un lieu où tout peut se produire. Elle offre des tableaux aux multiples couleurs et met en présence personnages et animaux. La nature, la forêt sont un refuge, c'est pourquoi, dans ce conte, les machines interdisent aux habitants d'y accéder: ils ne peuvent, en aucun cas, ressentir le besoin d'une existence plus humanisée.

De fait, sur l'île tout sentiment, que ce soit l'amour, l'espoir ou le désir, est banni, pour que les machines puissent maintenir leur contrôle sur les habitants et, de la sorte, éviter toute rébellion de leur part. Dans ce sens, les robots interdisent l'accès aux lieux qui, par la promenade, peuvent inspirer le sentiment de liberté, voire même permettre de côtoyer le reste de la civilisation humaine qui existe encore sur l'île. Par conséquent, ce lieu hors du temps, où règnent l'ordre et la loi dictés par les machines, ressemble davantage à une descente aux enfers, les habitants soumis à ce pouvoir considérant " [qu']il n'y a rien de mieux que leur gouvernement" (Dhôtel, 2002: 66). Bien que les habitants de l'île respectent et acceptent la domination des machines sans contestation, une atmosphère déplaisante envahit tout le récit, puisque nous découvrons que leurs gestes sont minutieusement contrôlés et leur vie menacée dès qu'ils ont un geste suspect.

Rappelons, néanmoins, que lorsque Julien " [aperçoit] l'île à un mille vers le nord " " (Dhôtel, 2002 : 26), il est ébloui en présence de ce nouveau décor et il se sent plongé dans un univers mystérieux et féerique. "'Voici encore un mystère', pensa Julien " (Dhôtel, 2002: 53). Bien que le mystère soit une caractéristique essentielle de l'île - en tant qu'espace paradisiaque et idyllique - nous constatons, néanmoins, que cette île 
dhôtelienne, où vivent des oiseaux de fer, est un lieu néfaste à l'homme, puisqu'elle l'empêche de rêver, d'aspirer à un absolu.

Mais le fait de se sentir prisonnier va pousser le héros à raviver ses souvenirs, à revenir sur les moments passés auprès des siens. Le personnage se sent comme étouffé dans cet espace, de sorte qu'il en vient à se remémorer combien il était heureux et libre avant d'entreprendre ce voyage. Yves Reuter souligne que « ces lieux s'organisent, font système et produisent du sens " (Reuter, $1991: 55)$. Ainsi, en s'éloignant du lieu où il avait été heureux, en perdant ce bonheur, qui avait jusqu'alors été quelque peu oublié, notre héros, face à l'adversité ressentie sur cette île, prend finalement conscience que ce bonheur l'a toujours accompagné et se trouve où il n'a jamais cessé d'être: dans sa ville natale de Bermont.

L'̂̂le est donc, une fois de plus, une étape que le héros dhôtelien doit être capable de surmonter, en surpassant des épreuves telles que l'interdiction de parler, de penser, de ressentir, voire même d'agir librement. Julien considère donc que sa présence sur l'île doit être envisagée comme un " curieux séjour, dont il aurait plus tard l'occasion de faire des contes, quand il retournerait à Bermont» (Dhôtel, 2002:60), et c'est pourquoi il a hâte de repartir au point de départ de l'histoire, de revenir sur ses pas, de retrouver le réconfort de son foyer. Les épreuves subies, les obstacles surmontés, Julien prend conscience qu'il n'appartient pas à ce décor, à ce style de vie et que son bonheur, qu'il jugeait ailleurs, se trouve, en fait, où il n'a jamais cessé de se trouver : à Bermont.

Dans ce sens, Bernard Noël affirme que :

L'œuvre de Dhôtel est un lieu - autrement dit une création, parce qu'elle nous offre le lisible qui, à tous moments, est l'équivalent du visible. Cela signifie qu'au lieu d'imiter, de copier, de simuler, elle se contente d'offrir inlassablement à nos yeux ce qu'ils pourraient voir (Apud Blondeau, $2003: 271$ ).

Nous pouvons conclure que, bien que la présence du monde soit retracée par Dhôtel de manière sensible et privilégiée, la question de l'espace nous ouvre sur un ailleurs, sur une poétique du merveilleux sans que, pour autant, nous nous sentions désorientée. La subtilité de l'auteur fait que, au fil du temps de l'histoire, les lieux délimités soient le point de départ vers un monde ouvert, sur des horizons nouveaux. Fréquemment le héros dhôtelien en présence de ces lieux y subit de rudes épreuves, parce qu'effectuant un apprentissage initiatique, un cheminement conduisant du passage qui va de l'enfance ou de l'adolescence à celui de l'âge adulte. Ces espaces nous mènent sans cesse vers un ailleurs, parsemé d'événements insolites, où les personnages dhôteliens semblent vouloir faire suspendre le temps, dans l'attente du surgissement d'un fait ou d'une rencontre.

\section{BIBLIOGRAPHIE}

BACHELARD, Gaston (1978 [1957]). La Poétique de l'espace. Paris : PUF.

BLONDEAU, Philippe (2003). André Dhôtel ou les merveilles du romanesque. Paris : L'Harmattan. 
BLONDEAU, (2006). « Dhôtel citadin », in Cahiers André Dhôtel : Les lieux d'André Dhôtel. Paris : La route inconnue, pp. 95-102.

DHÔTEL, André (2002 [1956]). L'T̂le aux oiseaux de fer. Paris : Bernard Grasset, Les Cahiers rouges. GANNIER, Odile (2001). La Littérature de voyage. Paris : Ellipses.

MARIE, Charles P. (1998). « La signification du signe dans la rhétorique fabuleuse », in Colloque André Dhôtel - Angers 6 et 7 Décembre 1996. Angers : Presses de l'Université d'Angers, pp. 117-130.

PERRY, Edith (2006). « Les Romans insulaires d'André Dhôtel, L'T̂le aux oiseaux de fer et Ce lieu déshérité ", in Cahiers André Dhôtel : Les lieux d'André Dhôtel. Paris : La route inconnue, pp. 69-82. PIROTTE, Jean-Claude (1983). « Les Pays d'André Dhôtel », Magazine littéraire, pp. 80-85.

PROPP, Vladimir (1970). Morphologie du conte. Paris : Seuil.

ReUMAuX, Patrick (1984). L'Honorable Monsieur Dhôtel. Lyon : La Manufacture.

REUTER, Yves (1991). Introduction à l'analyse du Roman. Paris : Dunod, Bordas.

ROPARS-WUILLEUMIER, Marie-Claire (2002). Écrire l'espace. Saint-Denis : Presses Universitaires de Vincennes.

TODERO, Marianna Antonella (2006). « Dhôtel : le chercheur d'un nouvel espace », in Cahiers André Dhôtel : Les lieux d'André Dhôtel. Paris : La route inconnue, pp. 116-131.

VÉDRINE, Hélène (1990). Les Grandes conceptions de l'imaginaire de Platon à Sartre et Lacan. Paris : Librairie Générale Française.

VENOT PETITET, Monique (1998). « Dhôtel et l'absurde », in Colloque André Dhôtel - Angers 6 et 7

Décembre 1996. Angers : Presses de l'Université d'Angers.

WEISGERBER, Jean (1978). L’Espace romanesque. Lausanne : Éditions L'âge d'homme.

\section{RÉSUMÉS}

Bien que peu connu en dehors de la France, Dhôtel se voit attribuer de nombreux prix comme le Grand Prix de l'Académie Française, le Grand Prix National de Lettres, entre autres, et il laisse derrière lui une panoplie importante d'ouvrages : romans, contes pour enfants, poèmes, essais, préfaces, recueils de nouvelles, etc.. Nous nous proposons d'aborder la poétique dhôtelienne de l'espace dans le conte L'île aux oiseaux de fer, où se mélangent des lieux appartenant à l'expérience de vie de l'auteur et des endroits méconnus, un ailleurs aux multiples décors. Ainsi, dans cette œuvre en question, nous retrouvons des lieux géographiquement localisables, qui font partie du quotidien réel, des souvenirs de l'auteur, mais également des endroits inexplorés, inconnus, étranges et qui dépaysent quelque peu le lecteur, mais sans jamais faire en sorte qu'il se sente complètement perdu.

Although poorly known outside France, Dhotel was awarded multiple illustrious awards such as the Grand Prix de l'Académie Française, the Grand Prix National de Lettres, among others, and left behind a variety of important works: novels, stories for children, poems, essays, prefaces, collections of novels, etc. We propose to address the dhotelien poetic of space in the tale L'île aux oiseaux de fer, which blends places belonging to the author's life experience and unknown places, an elsewhere in multiple settings. Thus in the work in question, we find geographically localizable places, that are part of real everyday life, memories of the author, but also unknown, 
strange and unexplored places, that somewhat disorient the reader, but without ever making him feel completely lost.

INDEX

Mots-clés : imaginaire, ailleurs, Dhôtel (André), apprentissage, merveilleux

Keywords : imaginary, elsewhere, Dhôtel (André), learning, wonderful

\section{AUTEUR}

NATÁLIA GUILHERMINA OLIVEIRA LAMEIRAS ALVES

Université de Aveiro

natalia.alves[at]ua.pt 